\title{
Lentivirally Delivered Glial Cell Line-Derived Neurotrophic Factor Increases the Number of Striatal Dopaminergic Neurons in Primate Models of Nigrostriatal Degeneration
}

\author{
Stephane Palfi, ${ }^{1,2 *}$ Liza Leventhal, ${ }^{*}$ Yaping Chu, ${ }^{1}$ Shuang Y. Ma, ${ }^{1}$ Marina Emborg, ${ }^{1}$ Roy Bakay, ${ }^{5}$ \\ Nicole Déglon, ${ }^{3,4}$ Philippe Hantraye, ${ }^{2}$ Patrick Aebischer, ${ }^{3,4}$ and Jeffrey H. Kordower ${ }^{1}$ \\ ${ }_{1}^{1}$ Department of Neurological Sciences, Rush-Presbyterian-St. Luke's Medical Center, Chicago, Illinois 60612, \\ 2Commissariat à l'Energie Atomique, Centre National de la Recherche Scientifique, and Unité de Recherche Associée, \\ 2210 Service Hospitalier Frederic Joliot, and Commissariat à l'Energie Atomique, 94000 Orsay Cedex, France, ${ }^{3}$ Division \\ of Surgical Research and Gene Therapy Center, Lausanne University Medical School, 1015 Lausanne, Switzerland, 4The \\ Neurosciences Institute, Swiss Federal Institute of Technology, 1015 Lausanne, Switzerland, and ${ }^{5}$ Department of \\ Neurosurgery, Emory University, Atlanta, Georgia 30322
}

The primate striatum contains tyrosine hydroxylase (TH)immunoreactive (ir) neurons, the numbers of which are augmented after dopamine depletion. Glial cell line-derived neurotrophic factor (GDNF) strongly modulates the viability and phenotypic expression of dopamine ventral mesencephalic neurons. The effect of GDNF on TH-ir neurons intrinsic to the striatum has yet to be investigated. In the present study, stereological counts of $\mathrm{TH}$-ir striatal neurons in aged and parkinsonian nonhuman primates revealed that GDNF delivered via a lentiviral vector (lenti-) further increased the number of these cells. Aged monkeys treated with lenti-GDNF displayed an eightfold increase in $\mathrm{TH}$-ir neurons relative to lenti- $\beta$ galactosidase-treated monkeys. Unilateral 1-methyl-4-phenyl- 1,2,3,6-tetrahydropyridine treatment alone in young monkeys resulted in a bilateral eightfold increase in $\mathrm{TH}$-ir striatal cells. This effect was further magnified sevenfold on the side of lenti-GDNF treatment. These cells colocalized with the neuronal marker neuronal-specific nuclear protein. Some of these cells colocalized with GDNF-ir, indicating that an alteration in phenotype may occur by the direct actions of this trophic factor. Thus, GDNF may mediate plasticity in the dopamine-depleted primate brain, which may serve to compensate for cell loss by converting striatal neurons to a dopaminergic phenotype.

Key words: striatum; dopaminergic neurons; Parkinson's disease; primates; GDNF gene therapy; lentivirus
In contrast to lower species, the primate striatum contains a population of tyrosine hydroxylase (TH)-immunoreactive (ir) neurons whose function currently remains elusive. In this regard, few studies have focused on the role of intrinsic dopaminergic neurons within the adult primate striatum. Striatal TH-ir neurons are a subtype of interneurons distributed preferentially to the peripheral regions of the striatum. They were initially described in the nonhuman primate striatum by Dubach et al. (1987) and have been identified in the human striatum as well (Porritt et al., 2000). Their numbers have been reported to increase up to threefold in monkeys after dopaminergic denervation secondary to 1-methyl-4-phenyl-1,2,3,6-tetrahydropyridine (MPTP) toxicity (Betarbet et al., 1997). Double-label immunofluorescence studies revealed that this population of $\mathrm{TH}$-ir neurons colocalizes with glutamic acid decarboxylase (GAD), the rate-limiting enzyme for GABA. These TH-ir-positive cells are also immunoreactive for the dopamine transporter (DAT), indicating that they may be equipped for functional dopaminergic neurotransmission (Betar-

\footnotetext{
Received Dec. 4, 2001; revised March 21, 2002; accepted March 28, 2002.

This work was supported by a grant from the Department of Defense, a grant from the Parkinson's Foundation of the National Capital Area, and the Charles and M. V. Shapiro Foundation. We thank Theodora Kladis for expert histological assistance.

*S.P. and L.L. contributed equally to this work.

Correspondence should be addressed to Dr. Jeffrey H. Kordower, Department of Neurological Sciences, Rush Presbyterian Medical Center, 2242 West Harrison Street, Chicago, IL 60612. E-mail: jkordowe@rush.edu.

Copyright (ㄷ) 2002 Society for Neuroscience 0270-6474/02/224942-13\$15.00/0
}

bet et al., 1997). Confirming these experimental data, a recent clinicopathological investigation demonstrated that $\mathrm{TH}$-ir and DAT-ir striatal neurons are increased in number in Parkinson's disease (PD) (Porritt et al., 2000). Increases in the number of TH-ir striatal neurons in PD and after MPTP intoxication may be a means by which the adult striatum compensates for dopaminergic striatal denervation (Bezard and Gross, 1998). This suggests that the $T H$ gene that is normally quiescent in striatal neurons may be triggered under specific pathological conditions (Du et al., 1995).

If alterations in dopaminergic tone modulate striatal $\mathrm{TH}$-ir neuronal number, factors known to influence nigrostriatal circuitry may produce similar effects. Unequivocally, glial cell linederived neurotrophic factor (GDNF), a member of the transforming growth factor- $\beta$ superfamily, produces dramatic effects on dopaminergic nigrostriatal neurons (Lin et al., 1993; for review, see Collier and Sortwell, 1999). GDNF promotes the viability of, enhances the morphological differentiation of, and increases dopamine uptake by mesencephalic neurons in vitro and in vivo (Beck et al., 1995; Tomac et al., 1995; Choi-Lundberg et al., 1997; Collier and Sortwell, 1999). In addition, increased levels of GDNF produced by injections, infusions, or gene delivery promote cell viability and induce phenotypic changes in dopaminergic midbrain neurons of rats with 6-OHDA lesions as well as aged rhesus monkeys and monkeys treated with MPTP (Gash et al., 1996; Lapchak et al., 1997; Tseng et al., 1997; Kordower et al., 2000). These trophic effects appear to be selective for specific 
neuronal populations, because the viability and phenotypic expression of serotonergic or GABAergic neurons are not affected by GDNF.

To date, the role of GDNF on primate TH-ir striatal neurons has not been investigated in vivo. We published a study recently that examined the structural and functional effects of GDNF in primate models of dopamine insufficiency (Kordower et al., 2000). This study provided the opportunity to examine these unique tissues and to evaluate the effects of GDNF on striatal TH-ir neurons. The present study assessed whether gene delivery of this trophic factor could augment the number of dopaminergic striatal neurons in primate models of nigrostriatal degeneration. First, we confirmed that dopamine depletion in MPTP-treated nonhuman primates enhances the number of TH-ir striatal neurons. Then we found that in vivo gene delivery of GDNF using a lentiviral vector increased the number of TH-ir and DAT-ir striatal neurons in aged monkeys. Finally, gene delivery of GDNF dramatically increased the number of $\mathrm{TH}$-ir striatal neurons in MPTP-treated monkeys.

\section{MATERIALS AND METHODS}

Experiment 1: effect of 1-methyl-4-phenyl-1,2,3,6-tetrahydropyridine on striatal tyrosine hydroxylase-immunoreactive cells. This experiment was performed to confirm previous findings now using stereological counting procedures to show that MPTP-treated monkeys have increased numbers of TH-ir striatal neurons (Betarbet et al., 1997). Four rhesus monkeys (4-5 years of age) received two unilateral intracarotid injections of MPTP separated by 1 month according to a protocol described previously (Kordower et al., 2000) (Table 1). Briefly, animals were tranquilized with ketamine $(10 \mathrm{mg} / \mathrm{kg}$, i.m. ) and then maintained on an anesthetic plane with isoflurane $(1-2 \%)$. The animals were put in the supine position. For each injection, a right-sided incision was made along the medial edge of the sternocleidomastoid muscle. The carotid sheath was opened and the common carotid artery, internal jugular vein, and vagus nerves were identified. The common carotid was exposed below the carotid bifurcation. The external carotid artery was then ligated. A 27 gauge butterfly needle was inserted into the common carotid artery in a direction retrograde to blood flow; for each injection, $20 \mathrm{ml}$ of saline containing $3 \mathrm{mg}$ of MPTP-HCl was infused at a rate of $1.33 \mathrm{ml} / \mathrm{min}(15$ $\mathrm{min}$ ). After the infusion was completed, $3 \mathrm{ml}$ of saline was delivered, and then the incision was closed. Two additional untreated rhesus monkeys served as unoperated controls.

Experiment 2: lentiviral delivery of glial cell line-derived neurotrophic factor to aged monkeys. This experiment investigated the effects of in vivo GDNF gene delivery on the number of striatal TH-ir cells in aged rhesus monkeys. The details of procedures performed on these monkeys have been reported previously (Kordower et al., 2000). In this experiment, four aged rhesus monkeys (24-27 years of age) received intrastriatal injections of lentivirus encoding for GDNF, and three monkeys received control injections of lentivirus encoding for $\beta$-galactosidase $(\beta$-gal) (Table 1). All animals received six lentivirus injections targeted for the caudate nucleus ( $n=2$ injections; 5 and $10 \mu \mathrm{l})$, putamen $(n=3 ; 10,10$, and $5 \mu \mathrm{l})$, and substantia nigra $(n=1 ; 5 \mu \mathrm{l})$. Coordinates were based on magnetic resonance imaging as described previously (Kordower et al., 2000). Monkeys were killed 3 months after the surgery. The construction of the vectors has been described previously (Kordower et al., 1999, 2000;

Table 1. Experimental groups

\begin{tabular}{lcc} 
& Group 1 & Group 2 \\
\hline Experiment 1: Effect of MPTP & MPTP & Intact control \\
on striatal TH-ir cells & $(n=4)$ & $(n=2)$ \\
Experiment 2: GDNF lentivi- & Aged/lenti-GDNF & Aged/lenti- $\beta$-gal \\
rus in aged monkeys & $(n=4)$ & $(n=3)$ \\
Experiment 3: GDNF lentivi- & & \\
rus in MPTP-treated & MPTP-lenti-GDNF & MPTP-lenti- $\beta$-gal \\
monkeys & $(n=4)$ & $(n=4)$
\end{tabular}

Deglon et al., 2000). Three months after the lentivirus treatment, all animals were killed.

Experiment 3: lentiviral delivery of glial cell line-derived neurotrophic factor to monkeys treated with 1-methyl-4-phenyl-1,2,3,6-tetrahydropyridine. This experiment tested the hypothesis that GDNF delivered via a lentiviral vector would increase the number of $\mathrm{TH}$-ir striatal neurons in MPTP-treated monkeys. Eight monkeys comprised this experiment, the methodological details of which have been reported previously (Kordower et al., 2000). Briefly, monkeys received a single injection of MPTP $(3 \mathrm{mg})$ into the right carotid artery followed 1 week later by injections of GDNF delivered by a lentiviral vector (lenti-) $(n=4)$ or lenti- $\beta$-gal $(n=$ 4) into the right caudate nucleus, putamen, and substantia nigra. The injection scheme was identical to that described above for aged monkeys. Three months after the lentivirus treatment, all animals were killed.

Preparation of tissues. Rhesus monkeys were killed with an overdose of pentobarbital, perfused transcardially with saline, and postfixed with $4 \%$ paraformaldehyde solution in phosphate buffer $(0.1 \mathrm{M}), \mathrm{pH} 7.4$, at $4^{\circ} \mathrm{C}$. The brains were removed immediately and cut into coronal blocks for postfixation. Tissue blocks were then washed in a series of cold graded sucroses and sectioned $(40 \mu \mathrm{m})$ in the coronal plane on a freezing microtome.

Immunohistochemistry. Sections through the forebrain and midbrain were processed for the visualization of TH, DAT, or GDNF immunoreactivity using modifications of procedures published previously (Kordower et al., 1999, 2000). Briefly, endogenous peroxidase activity was eliminated with a $20 \mathrm{~min}$ incubation in $0.1 \mathrm{M}$ sodium periodate. Background staining was then inhibited by a $1 \mathrm{hr}$ incubation in $3 \%$ normal serum and $2 \%$ bovine serum albumin (BSA), after which the tissue was incubated for $48 \mathrm{hr}$ in the primary antibody. The concentrations of the primary antibodies were as follows: TH (1:20,000; Chemicon, Temecula, CA), DAT (1:500; generously provided by Dr. Alan Levey, Emory University, Atlanta, GA), or GDNF (1:250; Transduction Laboratories, Lexington, KY). Using a labeled antibody procedure, sections were then sequentially incubated in the appropriate biotinylated $\operatorname{IgG}(1: 200$; Vector Laboratories, Burlingame, CA) for $1 \mathrm{hr}$, washed in a Tris-buffered saline solution containing $0.05 \%$ Triton X-100, and incubated in "Elite" avidinbiotin complex (ABC) (1:1000; ABC kits; Vector Laboratories) for 75 min. The chromogen solution that completed the reaction consisted of $0.05 \%$ diaminobenzidine and $0.005 \% \mathrm{H}_{2} \mathrm{O}_{2}$. For the GDNF staining, the immunohistochemical reaction product was intensified by adding $2.5 \%$ nickel II sulfate to the chromogen solution. Sections were mounted on gelatin-coated slides, dehydrated through graded alcohols (70, 95, and 99\%), cleared in xylenes, and coverslipped with Permount (Fisher Scientific, Pittsburgh, PA). Additional sections were processed simultaneously in an identical manner except for the deletion of the primary antibody or substitution of the primary antibody with an irrelevant IgG matched for protein concentration. No specific immunoreactivity was seen for any stain under these conditions.

Histological analysis. In experiment 1 , stereological counts of striatal TH-ir neurons were performed bilaterally on MPTP-treated animals and unilaterally in control animals. In experiments 2 and 3, stereological counts of TH-ir, GDNF-ir, or DAT-ir neurons were performed unilaterally on the side of the lenti-GDNF or lenti- $\beta$-gal infusions. For all experiments, stereological counts were performed using a computerized optical disector system. This method allows for an unbiased stereological quantification of a defined portion of a structure independent of its size, shape, orientation, tissue shrinkage, or anatomical level (Emborg et al., 1998). The computerized optical disector system consists of a computerassisted image analysis, a microscope, a computer-controlled $x, y, z$ motorized stage, a stereological software program (MicroBrightField Inc., Colchester, VT), and a high-sensitivity video camera. Before each series of measurements, the instrumentation was calibrated. The striatum was outlined under low magnification $(1.25 \times)$. Five percent of the outlined region was quantified using a disector counting frame and a systematic random-sampling design. The average section thickness for each section and the antibody penetration throughout the entire tissue section were determined empirically. The total number of striatal neurons was quantified using a high magnification $(100 \times)$ planapo oil immersion objective with a 1.4 numerical aperture. Under the disector principle, at least 200 striatal neurons were identified in each case. Once the top of the section was in focus, the $z$-plane was lowered 1-2 $\mu \mathrm{m}$. Care was taken to ensure that the top and bottom forbidden planes were not included in the analysis. The total number of TH-ir striatal neurons $(N)$ was calculated using the following formula: $N=N_{\mathrm{V}} \times V_{\text {Striatum, }}$, where 

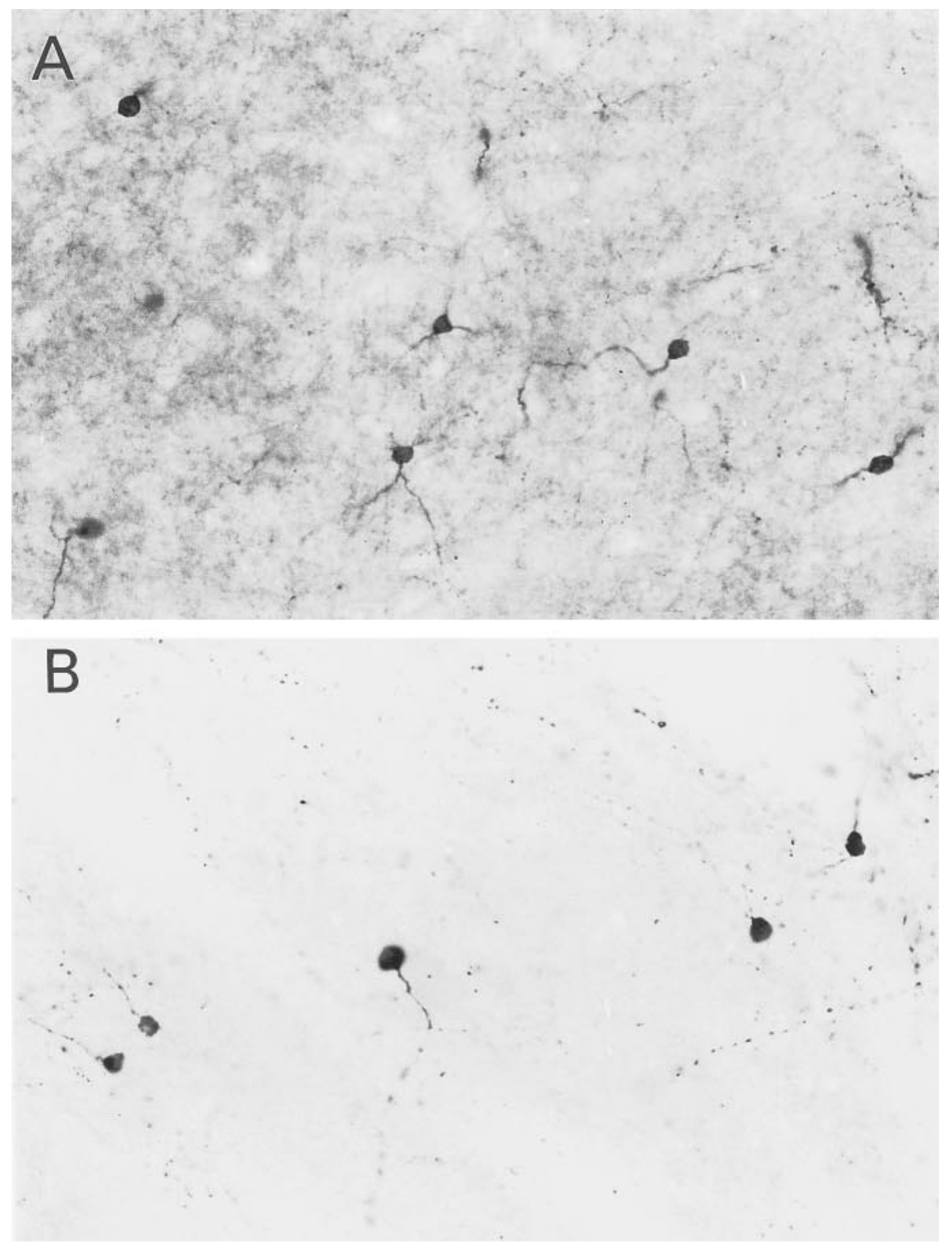

Figure 1. TH-immunostained sections through the striatum of a rhesus monkey receiving a unilateral intracarotid injection of MPTP $(A, B)$ and a normal control $(C)$. $A$, TH-ir neurons within the striatum on the side ipsilateral to the MPTP infusion. $B$, TH-ir neurons within the striatum on the side contralateral to the MPTP infusion. $C$, TH-ir neurons in an untreated rhesus monkey. Scale bar, $30 \mu \mathrm{m}$.

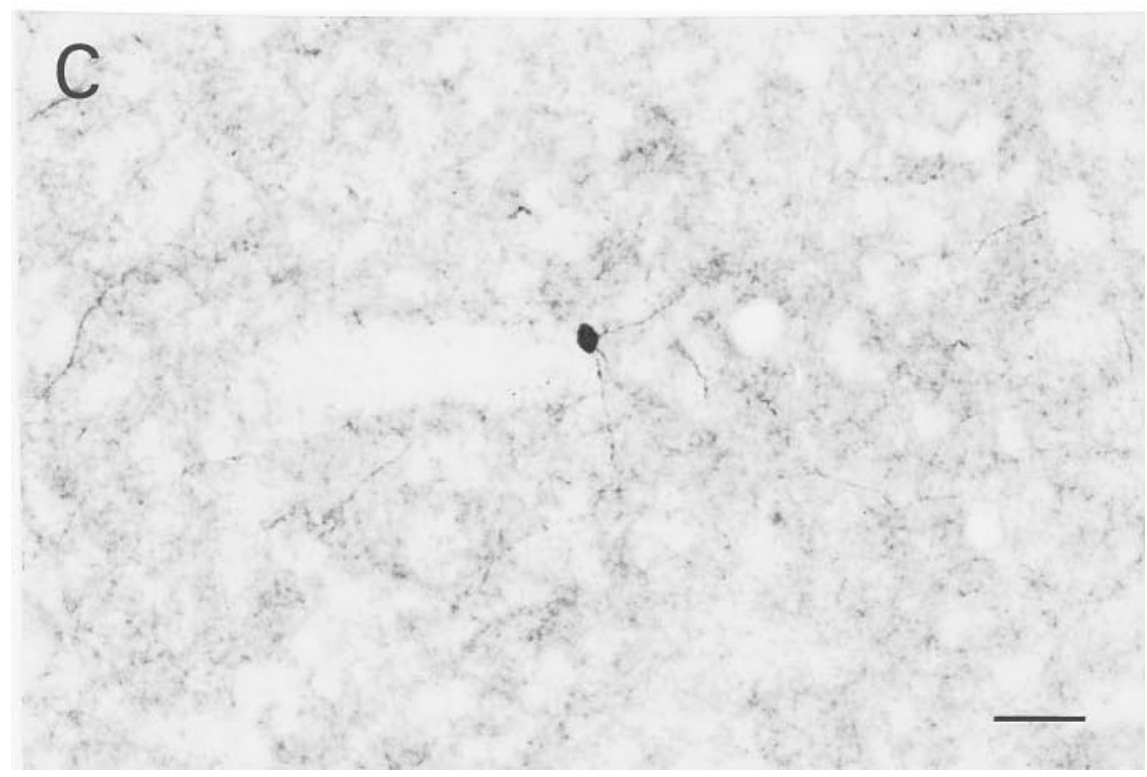


$N_{\mathrm{V}}$ is the numerical density and $V_{\text {Striatum }}$ is the volume of the striatum as determined by the Cavalieri principle (1966).

Confocal double immunofluorescence of tyrosine hydroxylase with neuronal-specific nuclear protein. Based on tissue availability, doubleimmunofluorescence experiments were performed with tissue from experiment 2 to determine whether the TH-ir striatal cells coexpressed the neuronal marker neuronal-specific nuclear protein $(\mathrm{NeuN})$ or the trophic factor GDNF. Sections were first incubated in a blocking solution (5\% normal goat serum, $2 \%$ BSA, and $0.3 \%$ Triton X-100 in TBS, pH 7.4) for $1 \mathrm{hr}$ to inhibit background staining. Then sections were incubated in primary rabbit polyclonal anti-TH $\left(1: 1000\right.$; Chemicon) for $24 \mathrm{hr}$ at $4^{\circ} \mathrm{C}$. After three washes, sections were incubated in secondary goat anti-rabbit IgG coupled to the fluorescent marker Cy2 (1:200; Jackson ImmunoResearch, West Grove, PA) for $1 \mathrm{hr}$. After completion of the reaction, sections were washed and background staining was inhibited by incubating the sections in a blocking solution containing $5 \%$ normal horse serum, $2 \% \mathrm{BSA}$, and $0.3 \%$ Triton $\mathrm{X}-100$ in TBS, $\mathrm{pH}$ 7.4. Then sections were incubated in mouse monoclonal anti-NeuN (1:1000; Chemicon) for $24 \mathrm{hr}$ at $4^{\circ} \mathrm{C}$ followed by incubation in goat anti-mouse $\mathrm{IgG}$ coupled to the fluorescent marker rhodamine (1:200, Jackson ImmunoResearch) for 1 $\mathrm{hr}$ at room temperature.

Confocal double immunofluorescence of tyrosine hydroxylase with glial cell line-derived neurotrophic factor. To assess double labeling of TH-ir cells with the neurotrophic marker GDNF, sections were immersed in the blocking solution as described above for $1 \mathrm{hr}$ and then incubated in primary mouse monoclonal anti-TH (1:10,000; Incstar, Stillwater, MN) for $24 \mathrm{hr}$ at $4^{\circ} \mathrm{C}$. After three washes, sections were incubated in secondary goat anti-mouse IgG coupled to the fluorescent marker Texas Red (1:200; Jackson ImmunoResearch) for $1 \mathrm{hr}$ at room temperature. Then sections were blocked with $5 \%$ normal horse serum, $2 \%$ BSA, and $0.3 \%$ Triton $\mathrm{X}-100$ in TBS, $\mathrm{pH} 7.4$, and incubated in goat polyclonal anti-GDNF (1:200; Chemicon) for $24 \mathrm{hr}$ at $4^{\circ} \mathrm{C}$ and in biotinylated secondary donkey anti-goat IgG (1:200; Vector Laboratories) coupled to the fluorescent marker Cy 2 for $1 \mathrm{hr}$ at room temperature. Statistical analysis. The data were analyzed using a two-way ANOVA followed by a Fisher's PLSD post hoc test when there was a significant overall ANOVA (Statview 4.0; Abacus Concepts, Calabasas, CA).

\section{RESULTS}

Experiment 1: effect of 1-methyl-4-phenyl-1,2,3,6tetrahydropyridine on striatal tyrosine hydroxylaseimmunoreactive cell number

In all animals, numerous TH-ir neurons were distributed within the nonhuman primate caudate nucleus and putamen. In normal control animals, these cells were preferentially distributed around the periphery of the striatum, just interior to surrounding white matter. In MPTP-treated monkeys, TH-ir striatal neurons were also distributed along the periphery of the striatum. However, unlike normal controls, they were abundantly distributed throughout the central portions of the caudate nucleus and putamen as well.

Greater numbers of TH-ir neurons were seen in MPTP-treated monkeys relative to normal controls (Figs. $1 A-C, 2$ ). In present study, we performed stereological counts to confirm previous findings (Betarbet et al., 1997) using nonstereological counting procedures. Stereological counts in the present study demonstrated that MPTP-treated monkeys have increased numbers of TH-ir striatal neurons. Statistical analyses revealed significant differences in TH-ir striatal neurons between treatment groups (factorial ANOVA; $F_{(2,7)}=21.2 ; p<0.001$ ) (Fig. 2). Monkeys with intracarotid MPTP injections displayed significant increases in the number of TH-ir cells ipsilateral to the MPTP injection (mean \pm SD, 1,557,526 \pm 137,769) compared with untreated controls $(453,528 \pm 137,769 ; p<0.004)$. Interestingly although the MPTP treatment was unilateral, the number of TH-irpositive striatal cells was also augmented on the side contralateral to MPTP treatment $(1,834,752 \pm 129,477)$. This number of TH-ir striatal neurons was statistically greater than that seen in control
MPTP INCREASES THE NUMBER OF TH-IR STRIATAL NEURONS IN MONKEYS

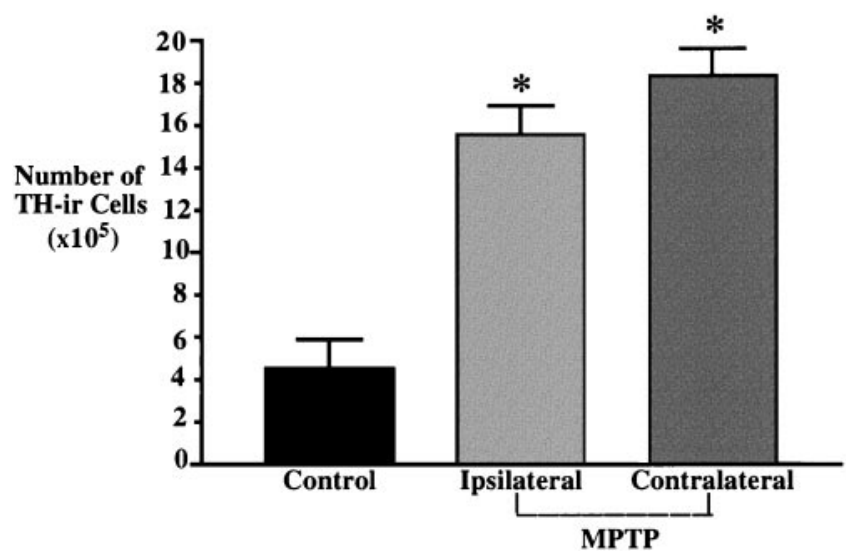

Figure 2. Histogram illustrating that the number of TH-ir-positive cells was significantly elevated ipsilateral and contralateral to the MPTP injection in monkeys receiving a unilateral intracarotid MPTP injection compared with the control nontreated animals. ${ }^{*} p<0.004$.

animals $(453,528 \pm 137,769 ; p<0.001)$ (Fig. 2) and similar in magnitude to that seen on the side ipsilateral to MPTP treatment $(p=0.90)$.

\section{Experiment 2: lentiviral delivery of glial cell line- derived neurotrophic factor in aged monkeys}

Lentiviral delivery of GDNF to aged monkeys resulted in a significant increase in striatal TH-ir and DAT-ir neurons compared with $\beta$-gal-treated control animals (Figs. 3, 4, 5, 6). The increase in the number of TH-ir striatal neurons was seen at every level of the striatum (Fig. $4 B, D, F$ ). In lenti- $\beta$-gal-treated animals, $\mathrm{TH}$-ir striatal neurons were scattered in the striatum but again were preferentially located within the lateral aspects of the caudate nucleus and putamen (Fig. 4A,C,E). In contrast, numerous $\mathrm{TH}$-ir striatal neurons were distributed through the center core of this structure in lenti-GDNF-treated animals (Fig. 4). Stereological counts through the striatum of aged monkeys receiving lentivirus encoding for GDNF revealed that the number of TH-irlabeled neurons was significantly augmented compared with lenti$\beta$-gal-treated cohorts (factorial ANOVA; $F_{(1,5)}=137.4 ; p<$ 0.0001) (Fig. 5A). Lenti-GDNF-treated aged monkeys displayed $2,851,744 \pm 179,249$ TH-ir cells compared with $350,009 \pm 28,105$ TH-ir striatal neurons in lenti- $\beta$-gal-treated animals. This represents a more than eightfold increase in the number of TH-ir cells (Fig. $5 A$ ). Similar significant increases mediated by lenti-GDNF were seen in DAT-ir neurons $\left(F_{(1,5)}=155.2 ; p<0.0001\right)$ (Figs. $5 C, 6)$. In lenti-GDNF-treated monkeys, 2,550,508 $\pm 143,991$ DAT-ir cells were seen in the striatum on the side ipsilateral to the lentivirus/MPTP inf usion. In contrast, only 379,625 \pm 43,649 DAT-ir striatal neurons were seen in Parkinsonian monkeys treated with lenti- $\beta$-gal (Fig. 5C). This represents a 6.7-fold increase in the number of TH-ir neurons.

GDNF-ir neurons were observed in aged monkeys treated with lenti-GDNF (Fig. 7 $A$ ) but not in aged moneys treated with lenti- $\beta$-gal (Fig. $7 B$ ). Stereological counts of GDNF-ir neurons were also performed (Fig. $5 B$ ). No GDNF-ir striatal neurons were seen in lenti- $\beta$-gal-treated animals, indicating that the injection schema used did not induce an upregulation of endogenous GDNF to within detectable levels. In contrast, 825,836 $\pm 13,466$ GDNF-ir neurons were seen within the striatum of lenti-GDNF- 


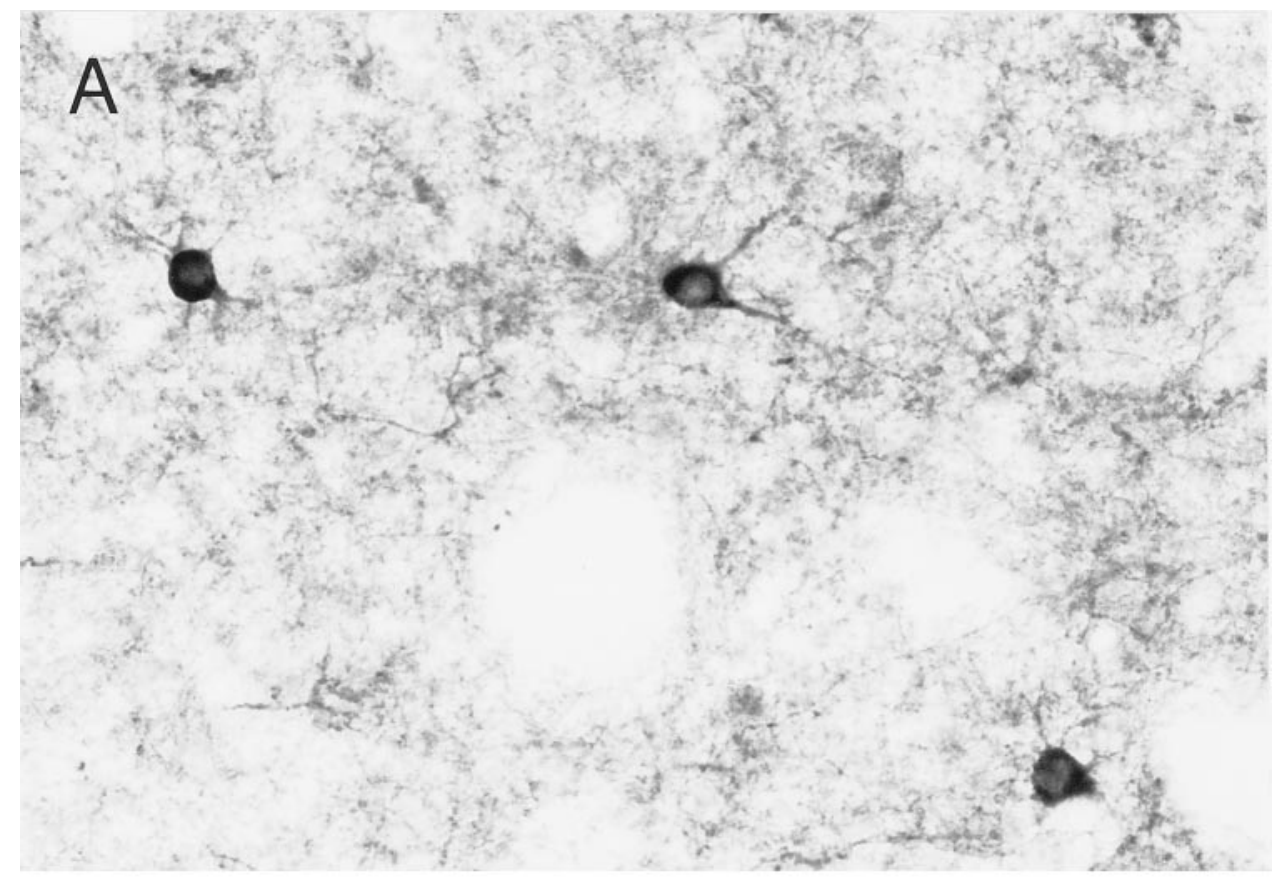

Figure 3. Photomicrographs of $\mathrm{TH}-$ immunostained sections through the striatum of aged monkeys receiving lentivirus encoding for $\operatorname{GDNF}(A)$ or $\beta$-gal $(B)$. Note that large and numerous TH-ir cells were observed in animals receiving lenti-GDNF compared with lenti- $\beta$-gal. Scale bar, $30 \mu \mathrm{m}$.

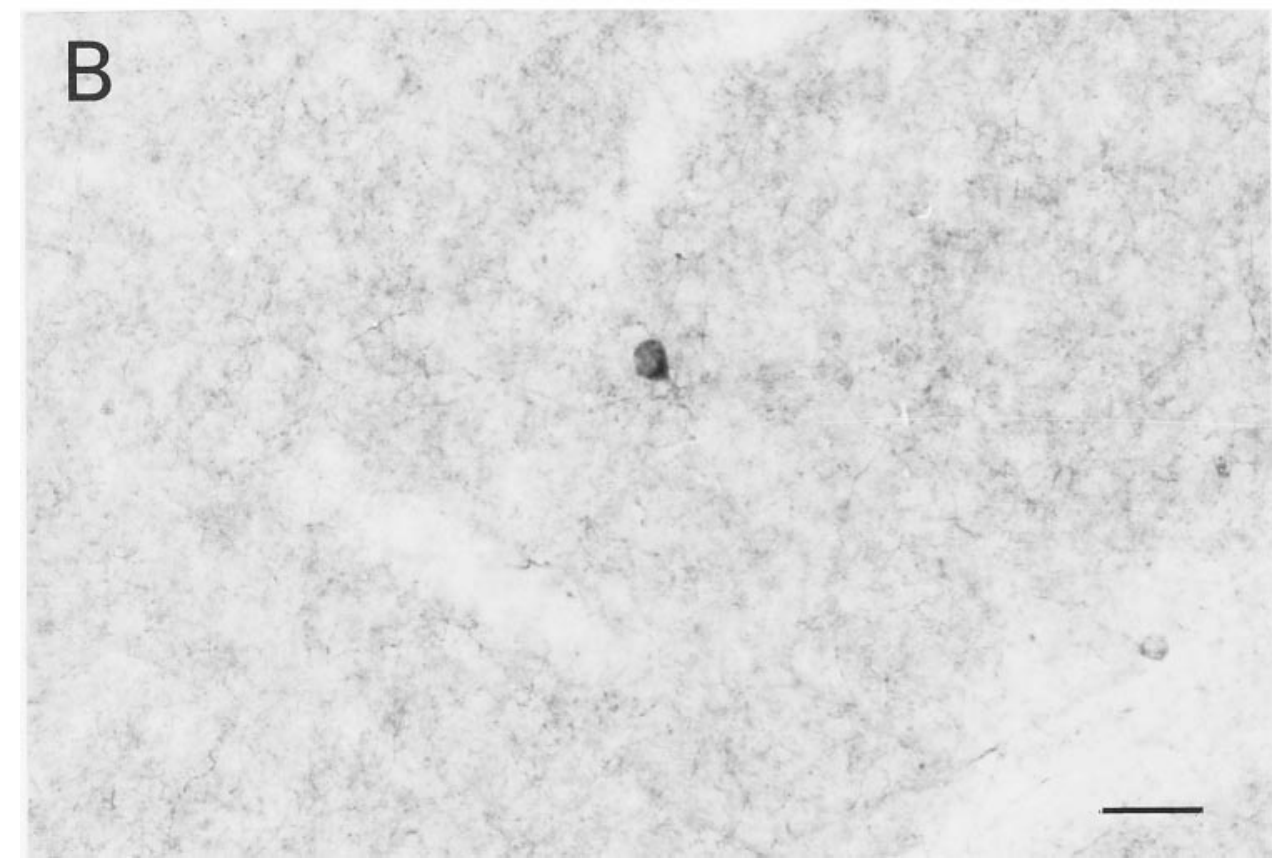

treated animals. Morphologically, the location of GDNF-ir cells varied depending on the site of lentiviral vector injections. In the penumbra of the injection site, GDNF-ir-positive cells appeared to be of similar size and displayed a similar morphology to that normally displayed by medium-sized spiny neurons. Additional tissues were not available to perform colocalization experiments to confirm that these cells are indeed medium spiny neurons. Robust extracellular GDNF staining was also observed in the penumbra of the injection site. In contrast, ovoid GDNF-irpositive cells were distributed primarily throughout the periphery of the injection area (Fig. $7 A$ ). Similarly, ovoid TH-ir- and DATir-positive cells were located in the same regions of the striatum as the ovoid GDNF-ir cells.

Correlations were performed to examine whether a significant association existed between GDNF-induced alterations of $\mathrm{TH}$-ir, GDNF-ir, and DAT-ir striatal cells. Significant correlations were observed between the various cell types: TH-ir/GDNF-ir $(r=$ 0.98; $p<0.0001)$, TH-ir/DAT-ir $(r=0.98 ; p<0.0001)$, and GDNF-ir/DAT-ir $(r=0.98 ; p<0.0001)$ (Fig. 5).

\section{Immunofluorescence}

Double-immunofluorescence experiments were performed to determine whether the TH-ir striatal cells coexpressed the neuronal marker NeuN or the trophic factor GDNF. Laser confocal microscopic images through the striatum of lenti-GDNF-treated monkeys confirmed that virtually all of the TH-ir cells colocalized with the neuronal marker NeuN (Fig. 8). These double-labeled cells were medium-sized and located in the penumbra of the 


\section{Distribution of TH-ir Cells \\ in Striatum of Aged Monkeys}

\section{Lenti-BGAL}

A.

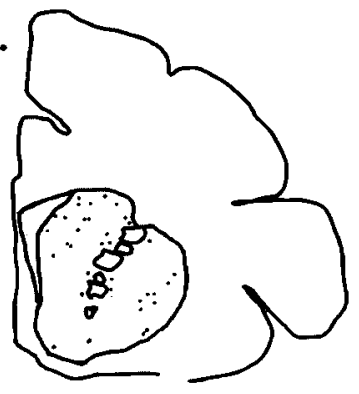

c.
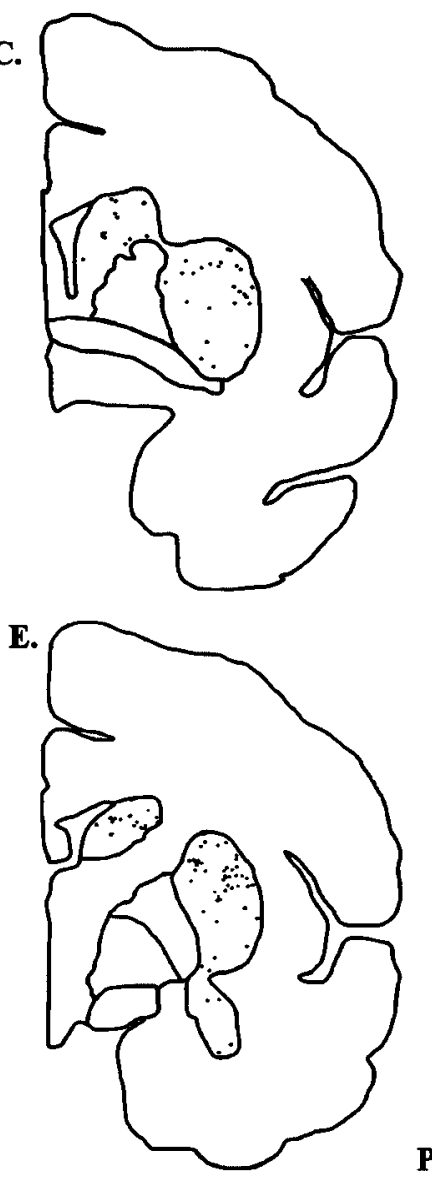

Precommissural

Commissural
B.

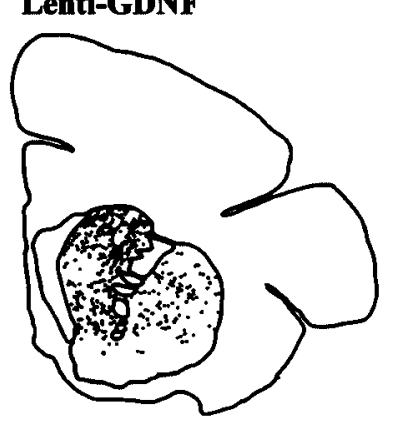

D.
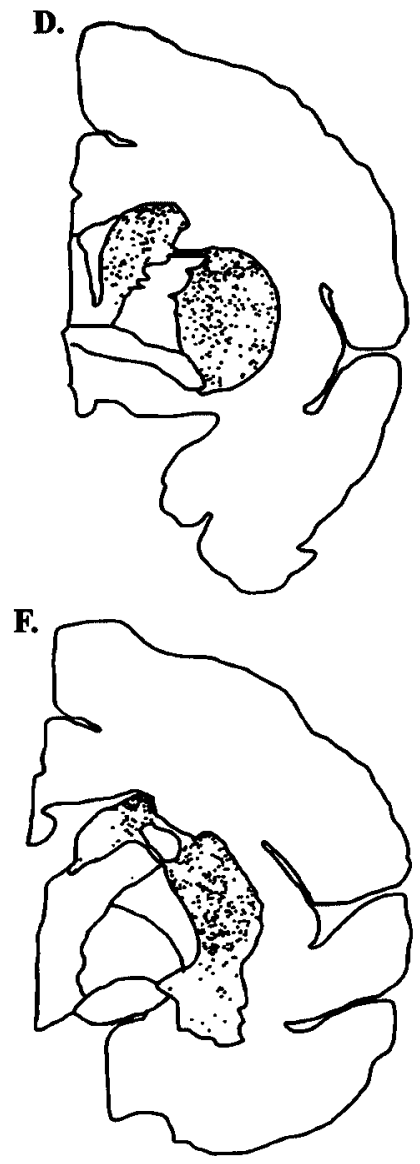

Figure 4. Distribution of TH-ir cells in the striatum of aged monkeys. Representative sections through the primate brain show the distribution of TH-ir cells throughout the precommissural $(A, B)$, commissural $(C, D)$, and postcommissural $(E, F)$ striatum of aged primates receiving intrastriatal injections of lentivirus encoding for $\beta$-gal $(A, C, E)$ or $\operatorname{GDNF}(B, D, F)$. Note that numerous TH-ir cells are distributed throughout the striatum in the lenti-GDNF-treated monkey compared with the lenti- $\beta$-gal-treated animal.
lenti-GDNF injection sites. Laser confocal microscopic images throughout the striatum of lenti-GDNF-treated monkeys indicated that numerous medium-sized neurons were immunopositive for GDNF and that the degree of colocalization between $\mathrm{TH}$ and GDNF was regionally specific. Few GDNF-ir cells located within the core of the injection site were immunoreactive for $\mathrm{TH}$ (Fig. 9). In contrast, many TH-ir-positive cells coexpressed GDNF-ir in regions distal to the transfection site. These doublelabeled cells displayed an ovoid shape. Again, no GDNF-ir immunofluorescence was observed in $\beta$-gal-treated animals.

\section{Experiment 3: lentiviral delivery of glial cell line- derived neurotrophic factor in monkeys treated with 1-methyl-4-phenyl-1,2,3,6-tetrahydropyridine}

In monkeys rendered parkinsonian with MPTP, lenti-GDNF augmented the number of $\mathrm{TH}$-ir striatal neurons by more than sevenfold relative to lenti- $\beta$-gal-treated animals (Fig. 10). In lenti$\beta$-gal-treated animals, stereological counts of $\mathrm{TH}$-ir neurons revealed $121,380 \pm 19,600$ positive cells on the treated side. In contrast, the striata of lenti-GDNF-treated animals contained $786,387 \pm 406,682 \mathrm{TH}$-ir-positive neurons. This difference in the number of TH-ir striatal neurons between lenti- $\beta$-gal- and lentiGDNF-treated animals was statistically significant $\left(t_{(1,6)}=3.267\right.$; $p<0.017)$.

\section{DISCUSSION}

The present series of experiments demonstrated that the number of TH-ir striatal cells in nonhuman primates increases in response to dopaminergic depletion; this effect is enhanced by the lentiviral delivery of GDNF. Double-labeling studies in 

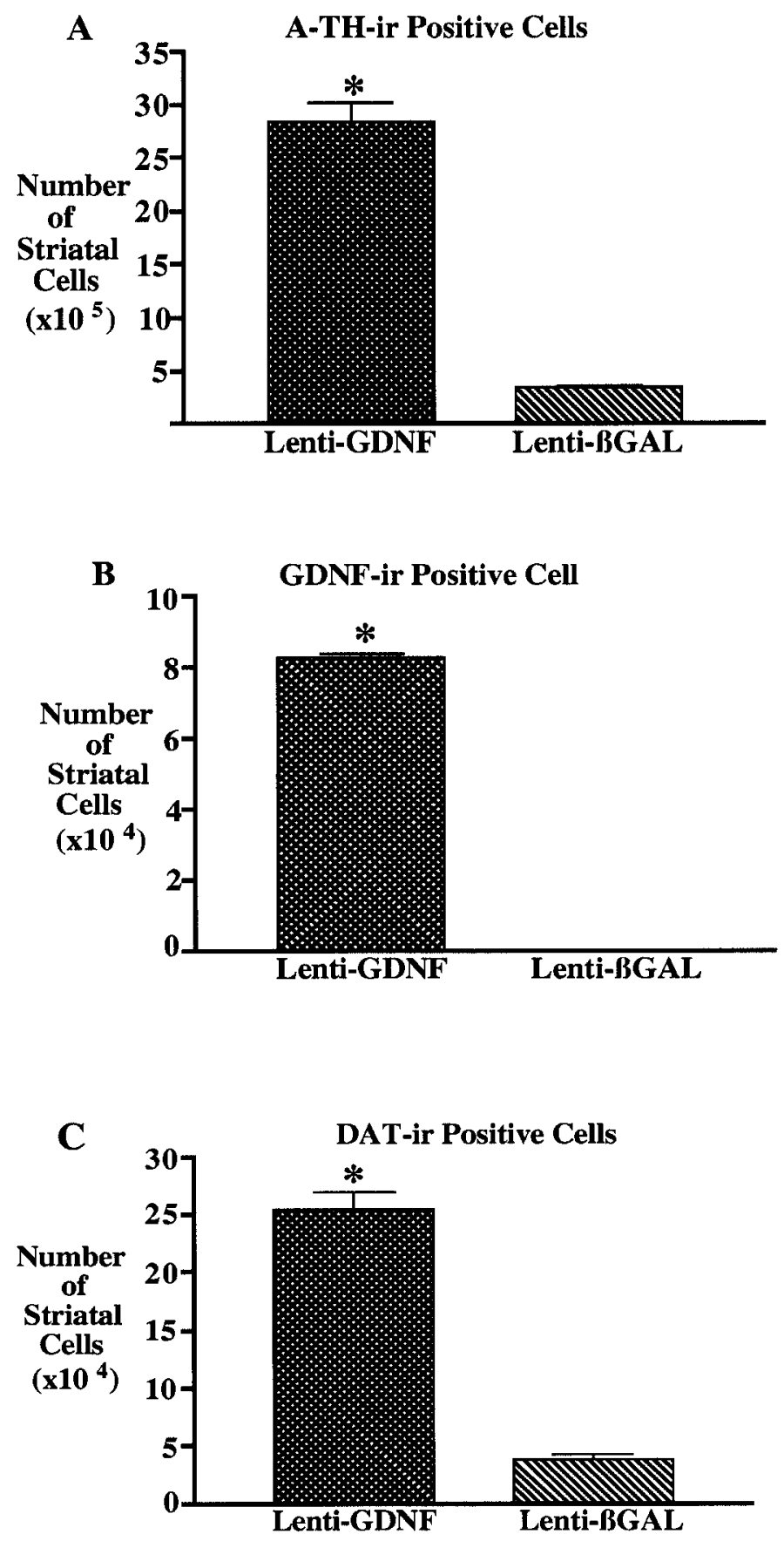
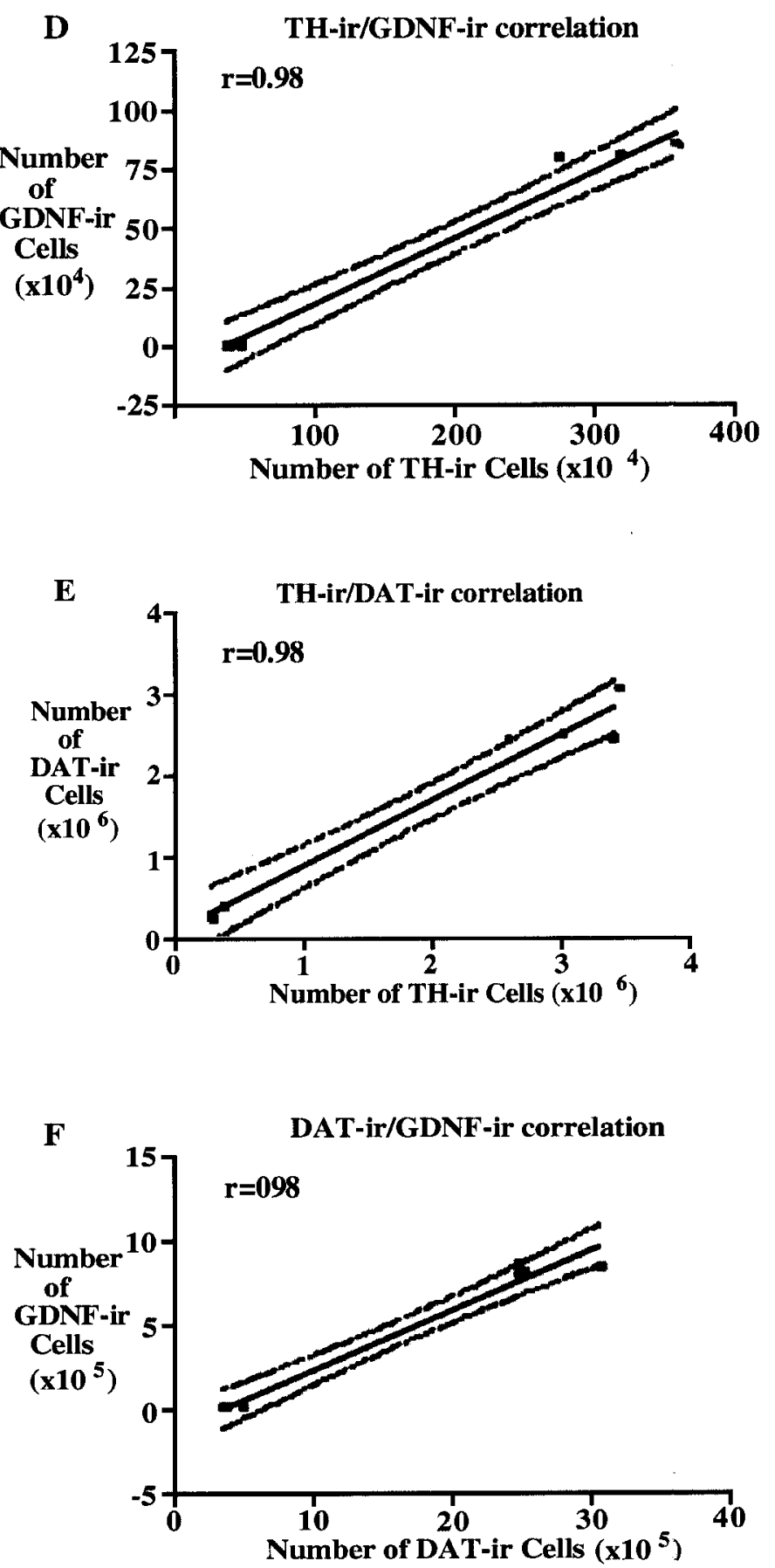

Figure 5. Histograms showing the number of TH-ir $(A)$, GDNF-ir $(B)$, and DAT-ir $(C)$ cells in the striatum of aged monkeys receiving lentivirus encoding for GDNF or $\beta$-gal. A significant increase of TH-ir $(A)$, GDNF-ir $(B)$, and DAT-ir $(C)$ cells was observed in animals receiving lenti-GDNF compared with the lenti- $\beta$-gal-treated cohorts. The correlation between the number of TH-ir/GDNF-ir, TH-ir/DAT-ir, and DAT-ir/GDNF-ir cells in aged monkeys receiving lentivirus encoding for GDNF or $\beta$-gal is shown in $D, E$, and $F$, respectively. In all cases, a significant correlation between cell types was observed $(p<0.0001)$. $* p<0.05$.

lentivirus-treated animals revealed that these TH-ir cells coexpressed NeuN-ir, indicating that cells expressing this dopaminergic marker were neurons. In addition, TH-ir neurons coexpressed GDNF-ir, indicating that the induction of the TH phenotype may be occurring via an autotrophic mechanism. It should be noted that the increase in DAT-ir cells after GDNF treatment was similar to that observed for TH-ir and that the numbers of $\mathrm{TH}$-ir and DAT-ir cells were strongly correlated. These data suggest that the effects of dopamine depletion and GDNF administration provide concomitant influences on the genes responsible for $\mathrm{TH}$ and DAT expression.

A previous study found increases in $\mathrm{TH}$-ir striatal neurons after the unilateral or bilateral nigrostriatal lesions engendered by MPTP in nonhuman primates (Betarbet et al., 1997). This study 

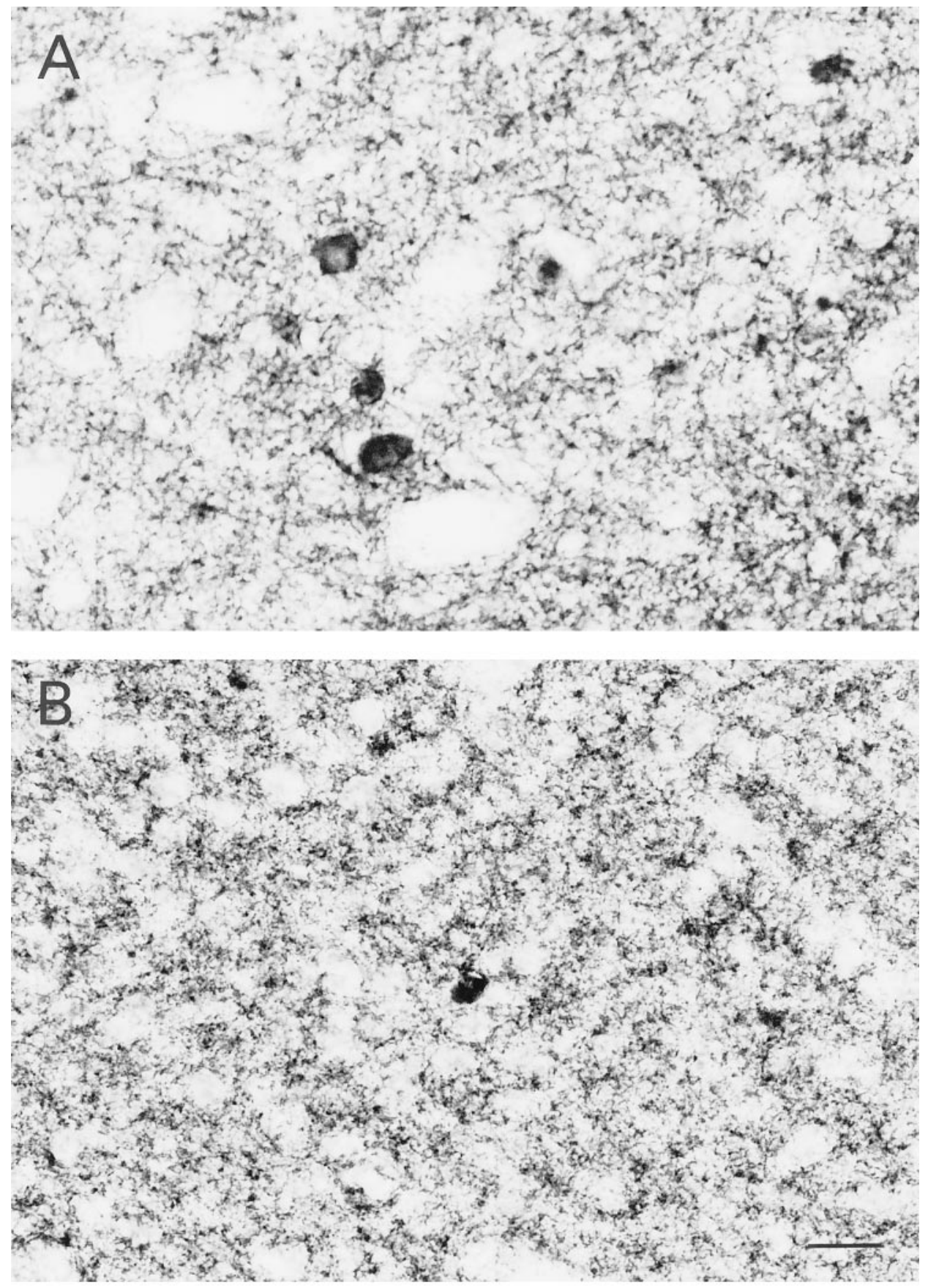

Figure 6. Photomicrographs of DAT-ir staining through the striatum of aged monkeys receiving lenti-GDNF $(A)$ or lenti- $\beta$-gal $(B)$. Note that large and numerous DAT-ir cells were observed in animals receiving lenti-GDNF compared with those receiving lenti- $\beta$-gal. Scale bar, $30 \mu \mathrm{m}$. quantified TH-ir neurons in four anatomically matched sections and did not have tissue prepared in a manner that was compatible with a stereological analysis. Stereological counts of TH-ir neurons in the present study confirmed the increase in neuronal number after MPTP-induced dopaminergic denervation. Indeed, in the study by Betarbet et al. (1997), bilateral increases in TH-ir striatal neurons were observed after unilateral MPTP injections. Stereological analyses in the present study confirmed this surprising effect as well. The fact that an increase in TH-ir striatal neurons was observed on the "intact side" strongly indicates that the increases seen on the side with lesions were authentic and not attributable to the fact that they were easier to identify and count after removal of the TH-ir striatal neuropil. However, it cannot be ruled out that the increase in $\mathrm{TH}$-ir striatal neurons seen on the intact side may have resulted from a small loss of nigrostriatal neurons that occurred as a result of MPTP crossing over to the contralateral side after the unilateral intracarotid injection of MPTP. In this regard, a small loss of TH-ir has been reported previously in monkeys on the side contralateral to the MPTP injection (Bankiewicz et al., 1999). If MPTP crossover mediated the increase in TH-ir neurons on the intact side, this would suggest that just a small loss of striatal dopamine is capable of eliciting this plasticity response.

It should be noted that the number of $\mathrm{TH}$-ir striatal neurons in the control MPTP/lenti- $\beta$-gal-treated animals in experiment 3 was less than what was observed in experiment 1 , in which monkeys were treated with MPTP alone. There are a number of factors that could have contributed to this difference in neuronal 


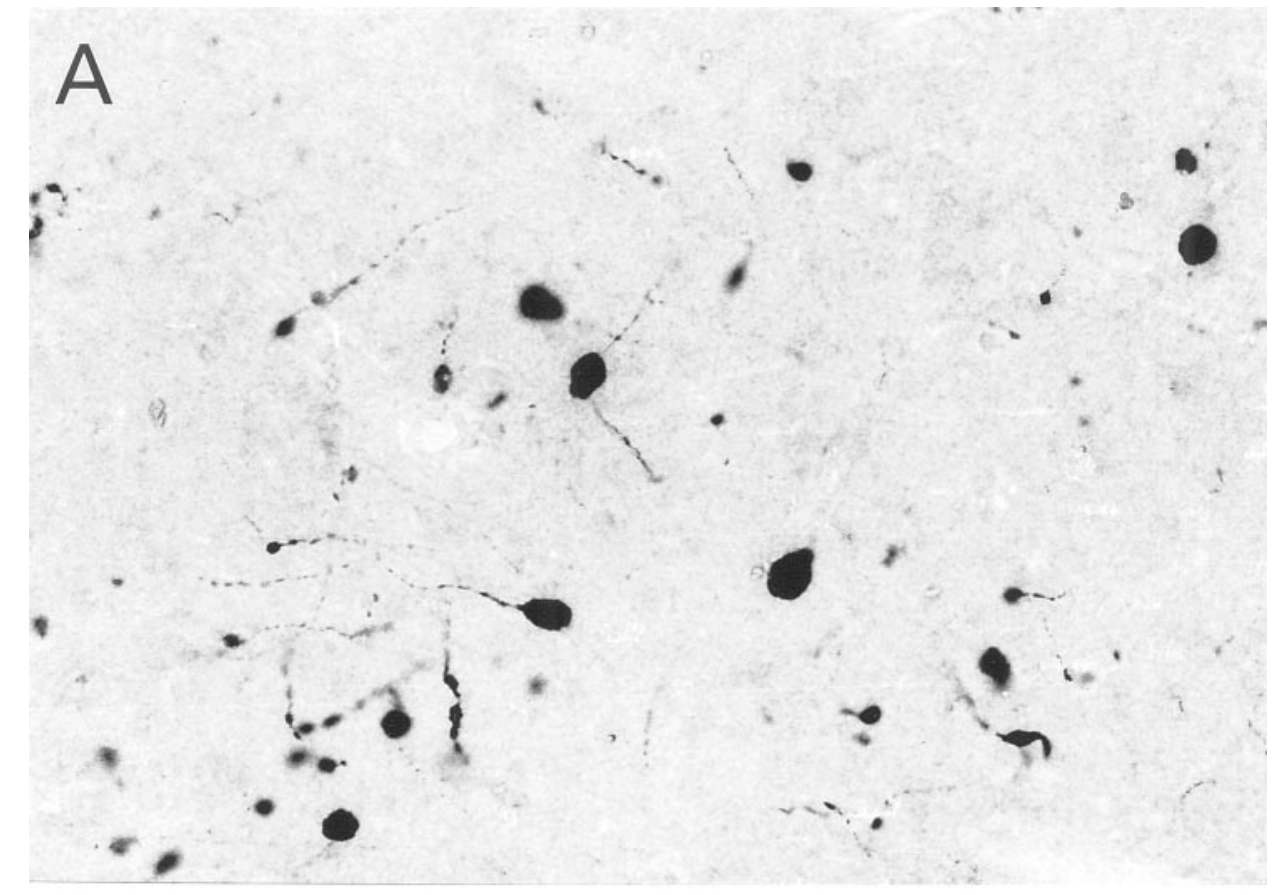

B

Figure 7. Photomicrographs of GDNF-ir in the striatum of aged monkeys receiving lenti-GDNF $(A)$ or lenti- $\beta$-gal $(B)$. Note that numerous GDNF-ir cells were observed in animals receiving lenti-GDNF, but no GDNF-ir-positive elements were seen in animals receiving lenti- $\beta$-gal. Scale bar, $30 \mu \mathrm{m}$.

number. In experiment 1 , the monkeys were exposed to multiple MPTP injections and were killed 5 months after surgery, whereas the monkeys in experiment 3 received a single MPTP injection and were killed 3 months postoperatively. Thus, repetitive injections combined with a longer postoperative time course could be responsible for this discrepancy, In addition, technical issues could have played a role in this difference. In each of the three experiments, tissue from control and experimental animals was histochemically processed together. However, tissues from the different experiments were processed independently, using different lots of antibody and different reagents. The intensity of staining was much greater in general through all dopaminergic regions in the tissues processed in experiments 1 and 2 compared with experiment 3; this likely contributed to the differences in absolute numbers. The critical comparisons to be concerned with in this study are the differences between conditions within an experiment rather than the absolute number of neurons responding to dopamine depletion and lenti-GDNF treatment.

The molecular mechanism underlying this plasticity response remains to be elucidated. Endogenous trophic factor release by glia in response to MPTP treatment has been reported as one potential stimulator of the normally quiescent $\mathrm{TH}$ gene (Francis et al., 1995) and may account for the increase in TH-ir cells. Regardless of whether this mechanism is in effect, the present data suggest that the increase of $\mathrm{TH}$-ir cells is a compensatory response to striatal dopamine depletion. However, the fact that nonhuman primates become symptomatic so quickly after intracarotid MPTP administration suggests that this response is insufficient to sustain functional effects in these animals.

In this study, lenti-GDNF dramatically increased the number 

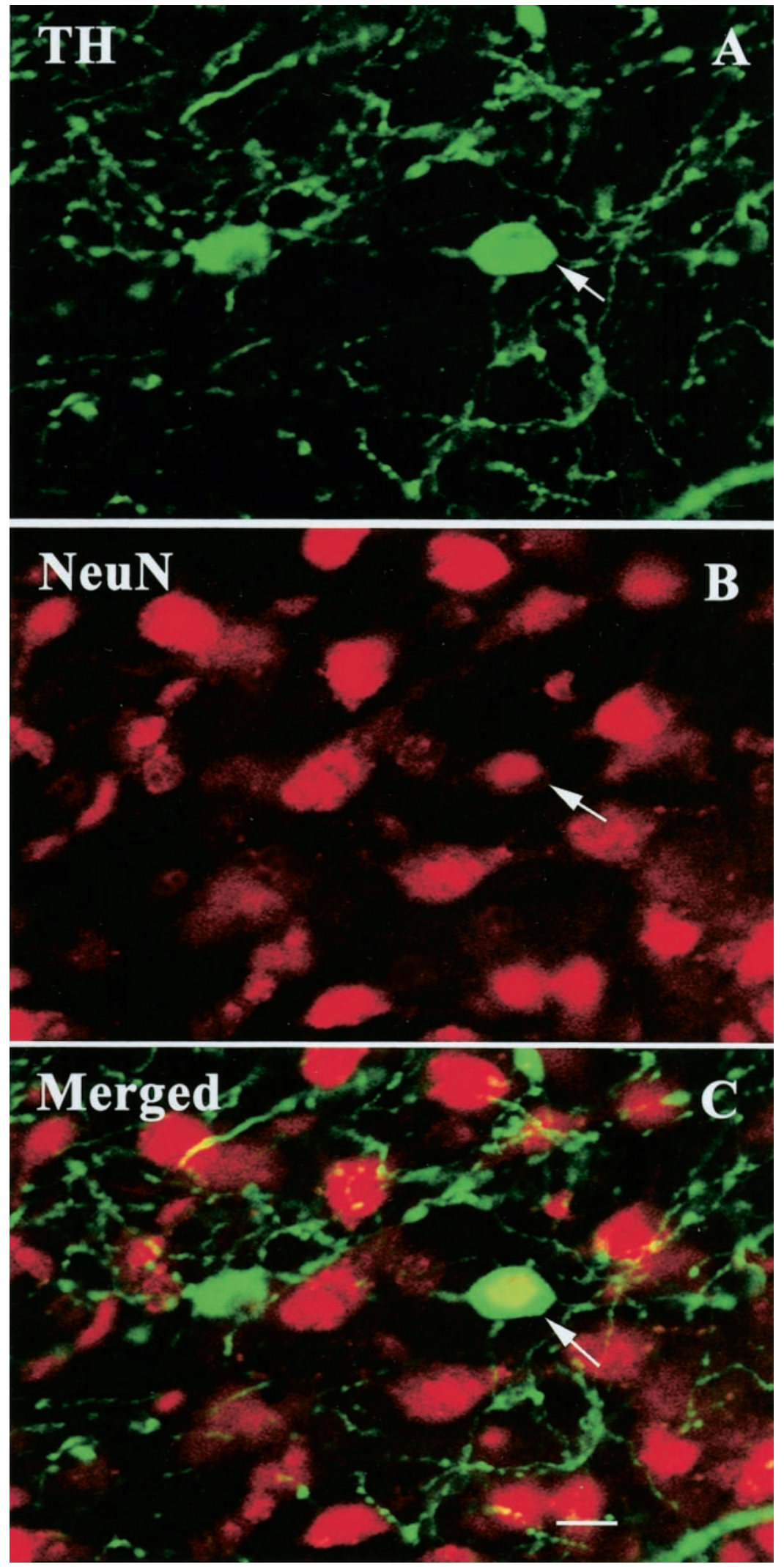

Figure 8. Laser confocal microscopic images through a series of focal planes through the caudate nucleus of an aged monkey treated with lenti-GDNF and stained for TH $(A)$ and NeuN $(B)$. $C$, The merged image. The yellow cells in $C$ signify that cells coexpress both $\mathrm{TH}$ and NeuN (arrow). The arrows depict the same cell in $A$ and $B$ as well as the merged image in $C$. Scale bar, $15 \mu \mathrm{m}$. of TH-ir striatal neurons in both aged monkeys and monkeys rendered parkinsonian via MPTP. Aged rhesus monkeys undergo phenotypic losses of nigrostriatal dopaminergic markers, as evidenced by the fact that $50 \%$ of nigral neurons fail to express $\mathrm{TH}$ and $30 \%$ fail to express DAT (Emborg et al., 1998). Intracarotid injection of MPTP induces a more robust and frank degeneration of nigrostriatal neurons, in which often up to $90 \%$ of nigrostriatal neurons are lost (Bankiewicz et al., 1999). It is interesting that 

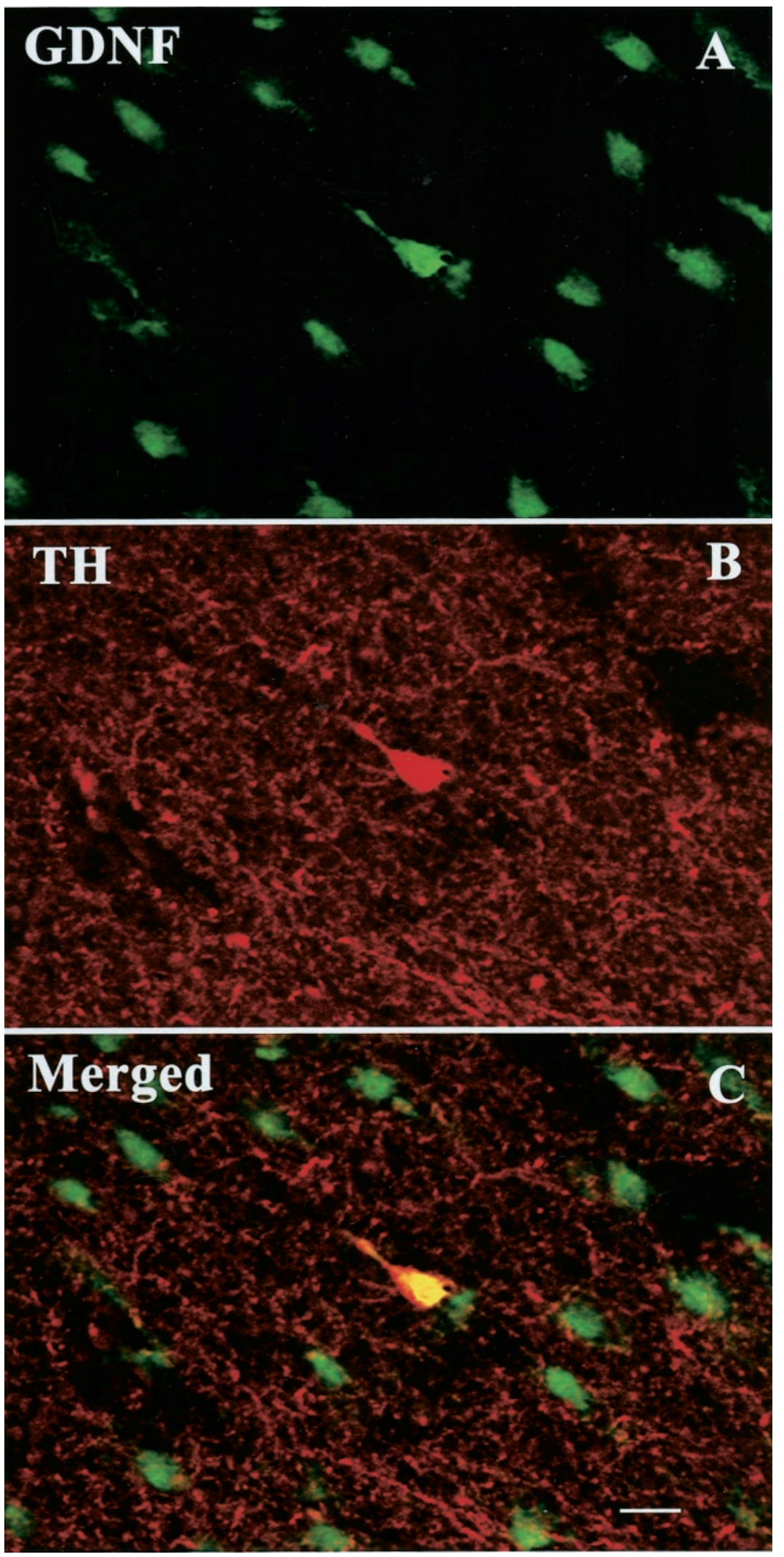

Figure 9. Laser confocal microscopic images through the caudate nucleus from an aged monkey treated with lentiGDNF. Green represents GDNF-ir; red represents TH-ir. Note the yellow cell in $C$, signifying that cells coexpress both GDNF and TH. Scale bar, $15 \mu \mathrm{m}$.

lenti-GDNF treatment was equipotent in increasing the number of TH-ir striatal neurons in MPTP-treated animals, in which the lesion was more severe relative to aged monkeys; in aged monkeys the lesion is more modest and does not produce frank neuronal degeneration. This suggests that the underlying status of the host system does not influence the ability of lenti-GDNF to modify the expression of $\mathrm{TH}$-ir striatal neurons.

The mechanism by which GDNF increases the number of 


\section{LENTI-GDNF INCREASES THE NUMBER OF TH-IR STRIATAL NEURONS IN MPTP-TREATED MONKEYS}

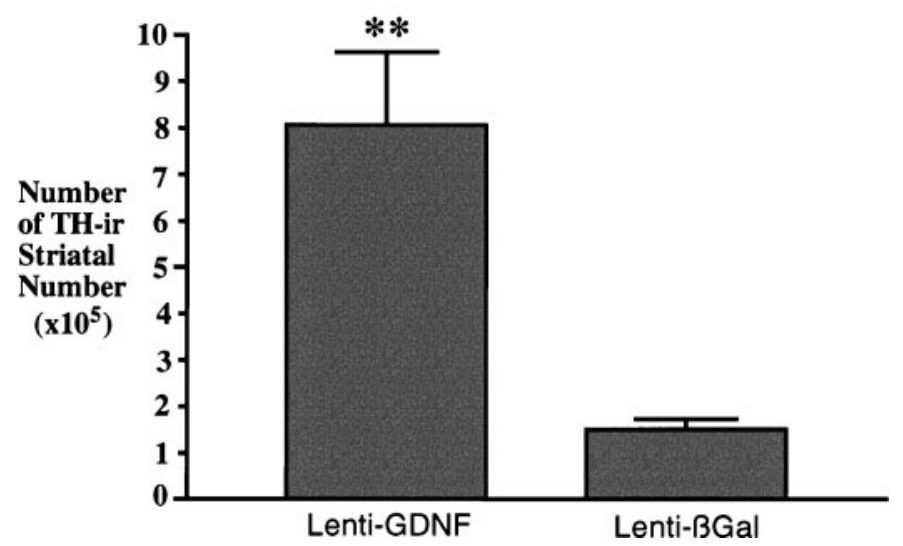

Figure 10. Histogram illustrating the dramatic increase in the number of TH-ir cells in the striatum of MPTP-treated monkeys receiving lentiGDNF or lenti- $\beta$-gal. ${ }^{* *} p<0.017$.

TH-ir neurons is not yet known. In the nigrostriatal dopaminergic system, GDNF has been shown to signal through a multireceptor complex composed of a novel glycosylphosphatidylinositol-anchored GDNF receptor and the receptor tyrosine kinase product of the c-ret proto-oncogene (Durbec et al., 1996; Trupp et al., 1996). The expression of this receptor complex is maximal during early postnatal development but it is at very low levels in the adult striatum (Trupp et al., 1997). As stated previously, the vast majority of TH-ir cells coexpressed GDNF-ir, suggesting that this trophic factor functioned in an autotrophic manner. Interestingly, there appears to be specificity in this response, because not all cells that were successfully transfected with the lentivirus expressed TH-ir. Unfortunately, sufficient tissue sections were not available to analyze the expression of ret and GDNF receptor $\alpha$ after GDNF delivery and to address some of these issues of mechanisms. Such studies are fertile ground for further investigation.

Some potential mechanisms that mediate the augmentation of striatal TH-ir neurons have been proposed. One hypothesis is that existing GABAergic striatal interneurons are converted to a TH-ir phenotype (Betarbet et al., 1997). This theory is based on a morphology analysis and colocalization of TH-ir with GAD-ir in the MPTP primate model of PD. In the present experiment, double labeling of TH-ir and GAD-ir were inconclusive (data not shown). One other potential mechanism may involve neural stem cells that migrate and differentiate to $\mathrm{TH}$-ir-positive neurons that result from dopamine depletion and/or trophic factor release (Svendsen and Smith, 1999). This later hypothesis has important implications for neural plasticity, but it needs to be confirmed experimentally.

In summary, the present study revealed that the number of TH-ir striatal cells in nonhuman primates increased in response to dopaminergic depletion; this effect was further magnified by GDNF treatment. In aged monkeys receiving lentivirus encoding for GDNF, many TH-ir cells coexpressed GDNF-ir and NeuN-ir staining, indicating that these neurons are converted to a phenotype directly by the trophic effects of
GDNF. In both aged and MPTP-treated monkeys, lentiGDNF increases the number of TH-ir neurons by more than sevenfold, illustrating the potency of this trophic factor for dopaminergic neurons other than those arising from the ventral midbrain. In this role, GDNF may mediate plasticity in the dopamine-depleted primate brain and may serve to compensate for cell loss by converting striatal neurons to a dopaminergic phenotype. Additional studies are needed to determine whether these TH-ir striatal cells are extant striatal neurons or are generated from differentiated progenitor cells, and whether TH-ir cells are capable of producing dopamine and exerting functional effects.

\section{REFERENCES}

Adolfsson R, Gottfries CG, Roos BE, Winblad B (1979) Post-mortem distribution of dopamine and homovanillic acid in human brain, variations related to age, and a review of the literature. J Neural Transm 45:81-105.

Bankiewicz KS, Sanchez-Pernaute R, Oiwa Y, Kohutnicka M, Cummins A, Eberling JL (1999) Animal models of Parkinson's disease. Methods Neurosci 9 4:1-32.

Beck KD, ValVerde J, Alexi T, Poulsen K, Moffat B, Vandlen RA, Rosenthal A, Hefti F (1995) Mesencephalic dopaminergic neurons protected by GDNF from axotomy-induced degeneration in the adult brain. Nature 373:339-341.

Betarbet R, Turner R, Chockkan V, Delong MR, Allers KA, Walters J, Levey AI, Greenamyre JT (1997) Dopaminergic neurons intrinsic to the primate striatum. J Neurosci 17:6761-6768.

Bezard E, Gross CE (1998) Compensatory mechanisms in experimental and human parkinsonism: towards a dynamic approach. Prog Neurobiol 55:93-116.

Calne DB, Langston JW (1983) Aetiology of Parkinson's disease. Lancet 2:1457-1459.

Carlsson A, Winblad B (1976) Influence of age and time interval between death and autopsy on dopamine and 3-methoxytyramine levels in human basal ganglia. J Neural Transm 38:271-276.

Castillo SO, Baffi JS, Palkovits M, Goldstein DS, Kopin IJ, Witta J, Magnuson MA, Nikodem VM (1998) Dopamine biosynthesis is selectively abolished in substantia nigra/ventral tegmental area but not in hypothalamic neurons in mice with targeted disruption of the Nurr1 gene. Mol Cell Neurosci 11:36-46.

Cavalieri B (1966) Geometri degli indivisibli Tornio, Unione Tipografico, Editrice, pp 1-543.

Chen EY, Kallwitz E, Leff SE, Cochran EJ, Mufson EJ, Kordower JH, Mandel RJ (2000) Age-related decreases in GTP-cyclohydrolase-I immunoreactive neurons in the monkey and human substantia nigra. J Comp Neurol 30:534-548.

Choi-Lundberg DL, Lin Q, Chang Y, Chiang YL, Hay CM, Mohajeri H, Davidson BL, Bohn MC (1997) Dopaminergic neurons protected from degeneration by GDNF gene therapy. Science 275:838-841.

Collier TJ, Sortwell CE (1999) Therapeutic potential of nerve growth factors in Parkinson's disease. Drug Aging 14:261-287.

Deglon N, Tseng JL, Bensadoun JC, Zurn AD, Arsenijevic Y, Pereira de Almeida L, Zufferey R, Trono D, Aebischer P (2000) Selfinactivating lentiviral vectors with enhanced transgene expression as potential gene transfer system in Parkinson's disease. Hum Gene Ther 11:179-190.

Du X, Stull ND, Iacovitti L (1995) Brain-derived neurotrophic factor works coordinately with partner molecules to initiate tyrosine hydroxylase expression in striatal neurons. Brain Res 680:229-233.

Dubach M, Schimdt R, Kundel D, Bowden D, Martin R, German DC (1987) Primate neostriatal neurons containing tyrosine hydroxylase: immunohistochemical evidence. Neurosci Lett 75:205-210.

Durbec P, Marcos-Gutierrez CV, Kilkenny C, Grigoriou M, Suvanto P, Wartiovaara K, Smith D, Ponder B, Costantini F, Saarma M, Sariola H, Pachnis V (1996) Glial cell line-derived neurotrophic factor signaling through the Ret receptor tyrosine kinase. Nature 381:789-792.

Emborg ME, Ma SY, Mufson EJ, Levey AI, Taylor MD, Brown WD, Holden JE, Kordower JH (1998) Age-related declines in nigral neuronal function correlate with motor impairments in rhesus monkeys. J Comp Neurol 401:253-265.

Francis JW, Visger JV, Markelonis J, Oh TH (1995) Neuroglial responses to the dopaminergic neurotoxicant 1-methyl-4-phenyl$1,2,3,6$-tetrahydropyridine in mouse striatum. Neurotoxicol Teratol 17:7-12. 
Gash DM, Zhang Z, Ovadia A, Cass WA, Yi A, Simmerman L, Russell D, Martin D, Lapchak PA, Collins F, Hoffer BJ, Gerhardt GA (1996) Functional recovery in parkinsonian monkeys treated with GDNF. Nature 380:252-255.

Kordower JH, Bloch J, Ma SY, Chu Y, Palfi S, Roitberg BZ, Emborg M, Hantraye P, Déglon N, Aebischer P (1999) Lentiviral gene transfer to the nonhuman primate brain. Exp Neurol 160:1-16.

Kordower JH, Emborg ME, Bloch J, Ma SY, Chu Y, Leventhal L, McBride J, Chen EY, Palfi S, Roitberg BZ, Brown WD, Holden JE, Pyzalski R, Taylor MD, Carvey P, Ling Z, Trono D, Hantraye P, Déglon N, Aebischer P (2000) Neurodegeneration prevented by lentiviral vector delivery of GDNF in primate models of Parkinson's disease. Science 290:767-773.

Lapchak PA, Miller PJ, Collins F, Jiao S (1997) Glial cell line-derived neurotrophic factor attenuates behavioral deficits and regulates nigrostriatal dopaminergic and peptidergic markers in 6-hydroxydopaminelesioned adult rats: comparison of intraventricular and intranigral delivery. Neuroscience 77:745-752.

Lin LFH, Doherty DH, Lile JD, Bektesh S, Collins F (1993) GDNF: a glial cell line-derived neurotrophic factor for midbrain dopaminergic neurons. Science 260:1130-1132.

Porritt MJ, Batchelor PE, Hughes AJ, Kalnins R, Donnan GA, Howells
DW (2000) New dopaminergic neurons in Parkinson's disease striatum. Lancet 9223:44-45.

Svendsen CN, Smith AG (1999) New prospects for human stem-cell therapy in the nervous system. Trends Neurosci 22:357-364.

Tomac A, Lindqvist E, Lin LFH, Ogren D, Young D, Hoffer BJ, Olson $\mathrm{L}$ (1995) Protection and repair of the nigrostriatal dopaminergic system by GDNF in vivo. Nature 373:335-339.

Trupp M, Arenas E, Fainzilber M, Nilsson A-S, Sieber BA, Grigoriou M, Kilkenny C, Salazar-Grueso E, Pachnis V, Arumäe U, Sariola H, Saarma M, Ibáñez CF (1996) Functional receptor for glial cell linederived neurotrophic factor encoded by the c-ret proto-oncogene product. Nature 381:785-789.

Trupp M, Belluardo N, Funaksohi H, Ibanez CF (1997) Complementary and overlapping expression of glial cell line-derived neurotrophic factor (GDNF), c-ret proto-oncogene, and GDNF receptor- $\alpha$ indicates multiple mechanisms to trophic actions in the adult rat CNS. J Neurosci 17:3554-3567.

Tseng JL, Baetge EE, Zurn AD, Aebischer P (1997) GDNF reduces drug-induced rotational behavior after medial forebrain bundle transection by a mechanism not involving striatal dopamine. J Neurosci 17:325-333. 